\title{
Education
}

\section{Steps to interpret an electrocardiogram for healthcare professionals}

\author{
Israel Gitangaza ${ }^{1,2}$ \\ ${ }^{1}$ Dalian Medical University, Dalian, China \\ ${ }^{2}$ House of Medical Skills and Knowledge with IsraelGi, Rwanda
}

This article discusses the easy way and steps to interpret an electrocardiogram (ECG). ECG is one of the hardest topics for medical students, young medical doctors, cardiac nurses and other health professionals, that is why this topic has been chosen in order to help them to understand how to interpret ECG easily.

Electrocardiogram (ECG or EKG) is a fundamental part of cardiovascular assessment (Fig. 1, 2), which is used in recording the electrical signal from the heart to check

\section{Step 1: Confirming patient details}

1. Confirm the name and date of birth of the patient matches the details on the ECG.

2. Check the date and time that the ECG was performed.

for different heart conditions (1).

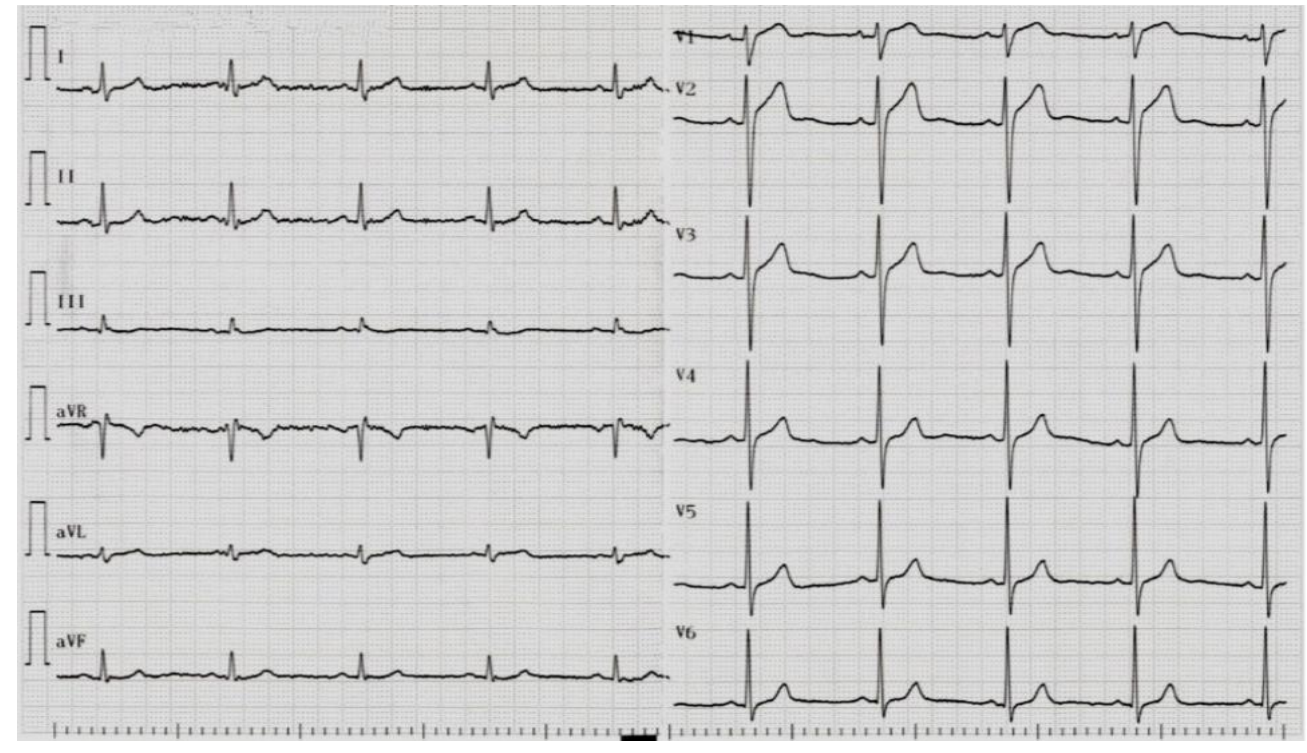

Figure 1. A 12-lead electrocardiogram recording

Address for Correspondence: Israel Gitangaza, House of Medical Skills and Knowledge with IsraelGi, Rwanda

Email: israelgitangaza@yahoo.com

Received: 03.03.2021 Revised: 02.05.2021 Accepted: 07.05.2021

Copyright $\bigcirc 2021$ Heart, Vessels and Transplantation 


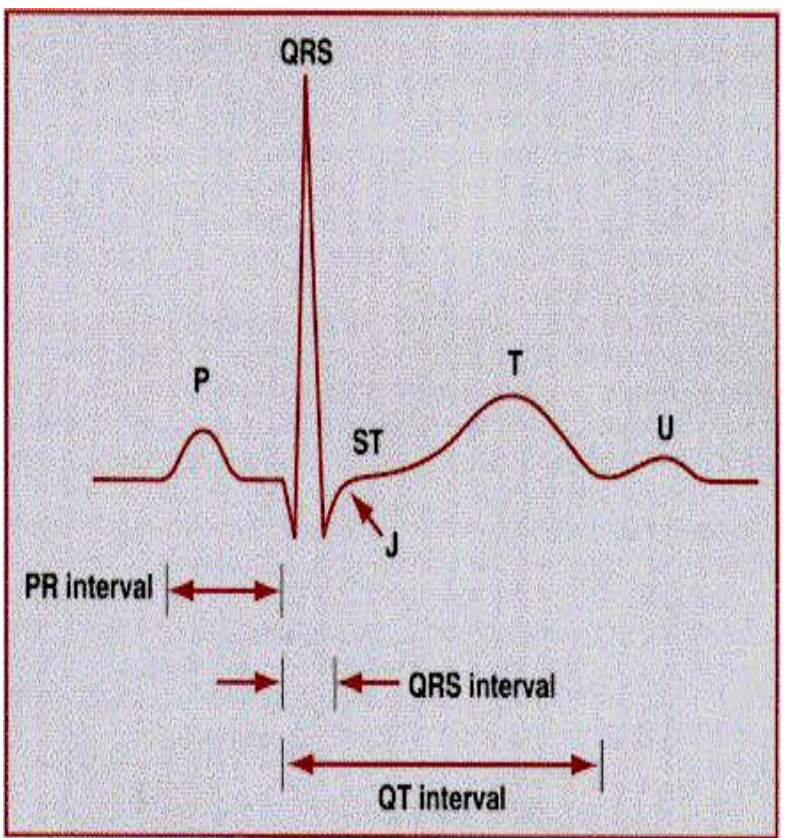

Figure 2. Normal electrocardiogram intervals, complexes and waves

\section{Step 2: Check ECG leads and check the aVR Lead}

ECG leads are obtained according to the localization of electrodes. Standard bipolar leads are recorded with one positive (+) and one negative (-) electrodes, whereas, unipolar leads are obtained with only one positive electrode. Three standard bipolar leads are I, II and III (Fig. 1). Among 3 bipolar leads in; lead I, positive electrode is placed on left arm, negative electrode is placed on right arm; lead II, positive electrode is placed on left leg, negative electrode is placed on right arm;lead III, positive electrode is placed on left leg, negative electrode placed on left arm .Unipolar leads are named according to the placement of positive electrode. If positive electrode is placed on right arm in aVR, left arm in aVL and left leg in AVF. In these 3 derivations voltage is low therefore special augmentation if performed. Because of this process lowercase ' $a$ ' letter is added ( $a=$ augmented) before the name of unipolar lead. I, II, III, aVR, aVL and aVF are named as standard extremity leads .
The unipolar chest (precordial) leads (V1, V2, V3, V4, V5 and $\mathrm{V6}$ ) have the exploring electrodes located anteriorly on the chest wall and the reference point located inside the chest (Fig. 1).

All waves should be positive (Fig. 2) apart from exception - the aVR lead. It is an unipolar lead facing the right superior surface. As all the depolarizations are going away from lead aVR, all waves are negative in aVR $(P, Q R S, T)$ in normal sinus rhythm. In dextrocardia, (true and technical) the $\mathrm{P}$ wave is upright in aVR (1).

\section{Step 3: Calculate and check heart rate}

Normal sinus rhythm rate: $50-100 \mathrm{bpm}$

Tachycardia: > 100 bpm

Bradycardia: < 50 bpm, sinus bradycardia: 40-50 bpm 


\section{I.How to calculate heart rate from ECG $(2,3)$ :}

\section{If a patient has a regular heart rhythm, his/her heart rate can be calculated using the following method:}

Count the number of large squares present within one

R-R interval.

Divide 300 by this number to calculate heart rate.

For example:

5 large squares in an $R-R$ interval $\boldsymbol{~} 300 / 5=60$ beats per

minute.

\section{If a patient has irregular rhythm}

Count the number of complexes on the rhythm strip (each rhythm strip is typically 10 seconds long). Multiply the number of complexes by 6 (giving you the average number of complexes in 1 minute).

For example: 10 complexes on a rhythm strip $\Rightarrow 10 \times 6=$ 60 beats per minute.

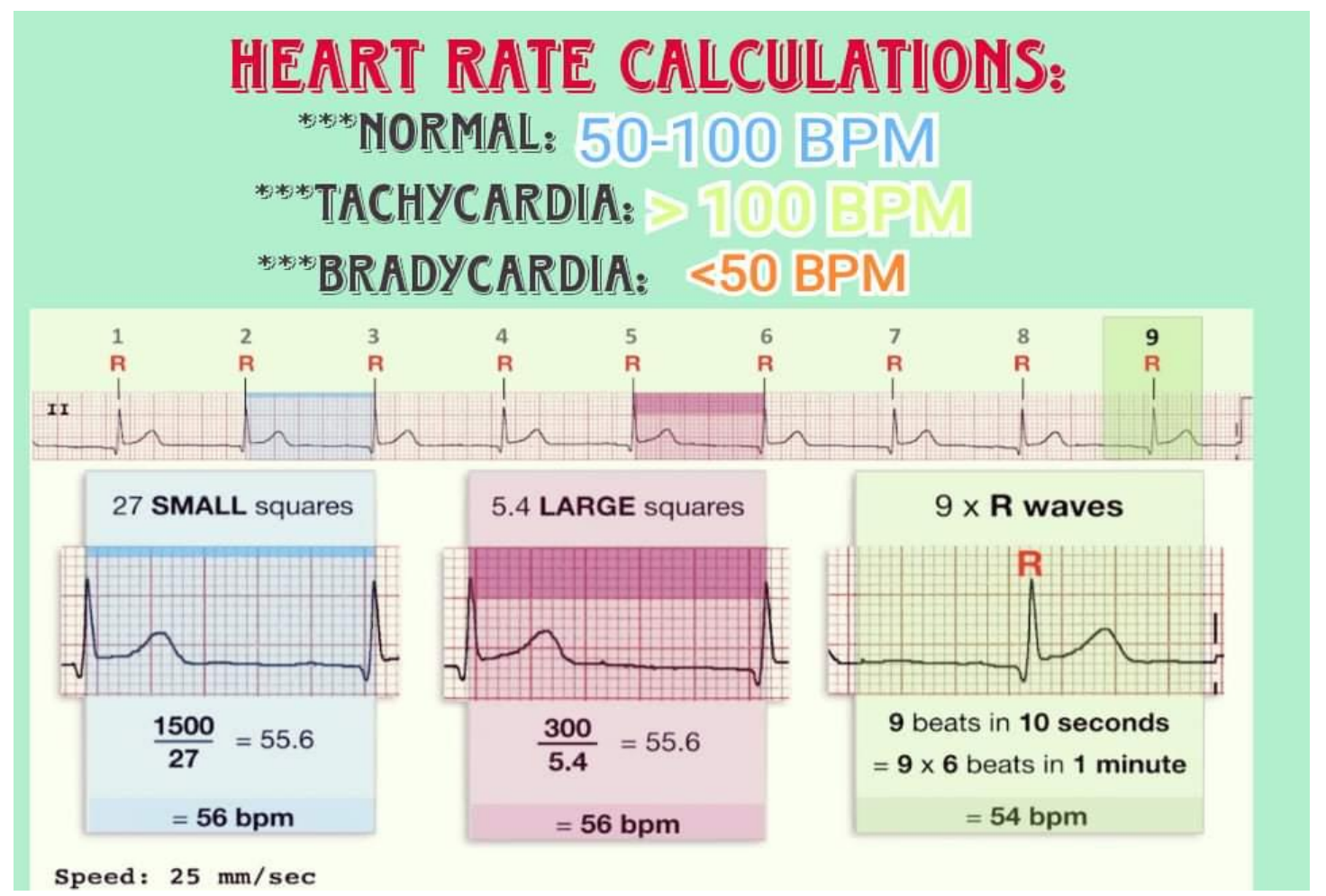

Figure 3. Heart rate calculations

\section{Step 4: Determine heart rhythm}

Normal cardiac rhythm, also known as sinus rhythm, arises from the sinoatrial node (SA node) but pacemaker impulses can come from ectopic foci in the atria, the atrioventricular (AV) junction, and the ventricles under abnormal conditions.

When an ectopic impulse occurs singly, it generates a beat; when the beat repeats itself, it becomes a rhythm (more than 3 beats).
Sinus rhythm implies that the SA node is the pacemaker and normal sinus rhythm (NSR) is simply sinus rhythm with heart rate in the normal range of 50-100 beats/min.

The $P$ waves in sinus rhythm have normal axis and are positive in lead II and negative in lead aVR. The QRS width in sinus rhythm is normal because the ventricles are activated rapidly by impulses conducted down the His bundle and bundle branches. Sinus bradycardia is a sinus rhythm with a rate of $40-50 \mathrm{bpm}$. Sinus tachycardia is a normal sinus rhythm but with a heart rate is over $100 \mathrm{bpm}$. 


\section{A patient's heart rhythm can be regular or irregular:}

I. Irregular rhythms can be either:

1. Regularly irregular: A rhythm is regularly irregular if it has some form or regularity to the pattern of irregular complex (i.e. a recurrent pattern of irregularity).
Example: Figure 4.1 - multifocal atrial tachycardia (MAT) in a patient with chronic obstructive pulmonary disease and adenocarcinoma of the lung. Three morphologically distinct P waves are shown with numbers in a lead II.

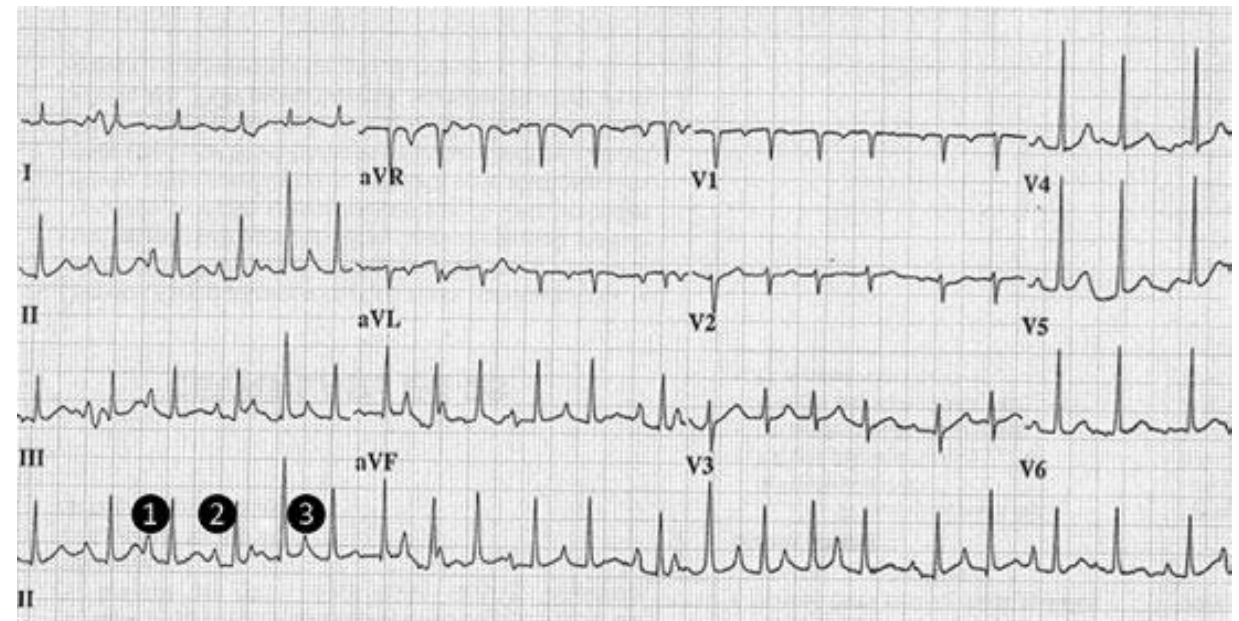

Fig 4.1. Multifocal atrial tachycardia (see text for explanation)

2. Irregularly irregular: A rhythm is irregular irregular if it has no pattern at all (i.e. completely disorganized). Mark out several consecutive R-R intervals on a piece of paper, then move them along the rhythm strip to check if the subsequent intervals are similar.

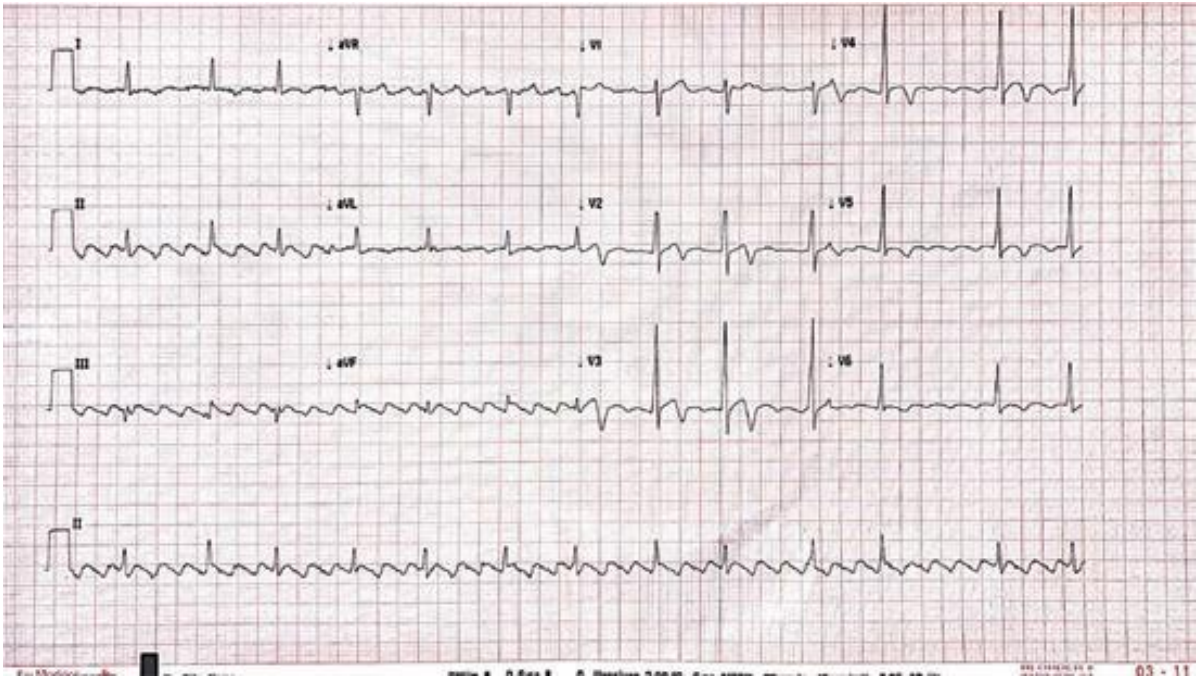

Figure 4.2. Atrial flutter
Example: Figure 4.2 Atrial flutter. ECG demonstrates atrial flutter with atrial rate of $300 \mathrm{bpm}$. Sawtooth pattern of flutter waves is seen in leads II, III, aVF. Ventricular rate is fixed and is fraction of atrial rate. There is $4: 1$ block and ventricular rate is $75 \mathrm{bpm}(2,3)$. 


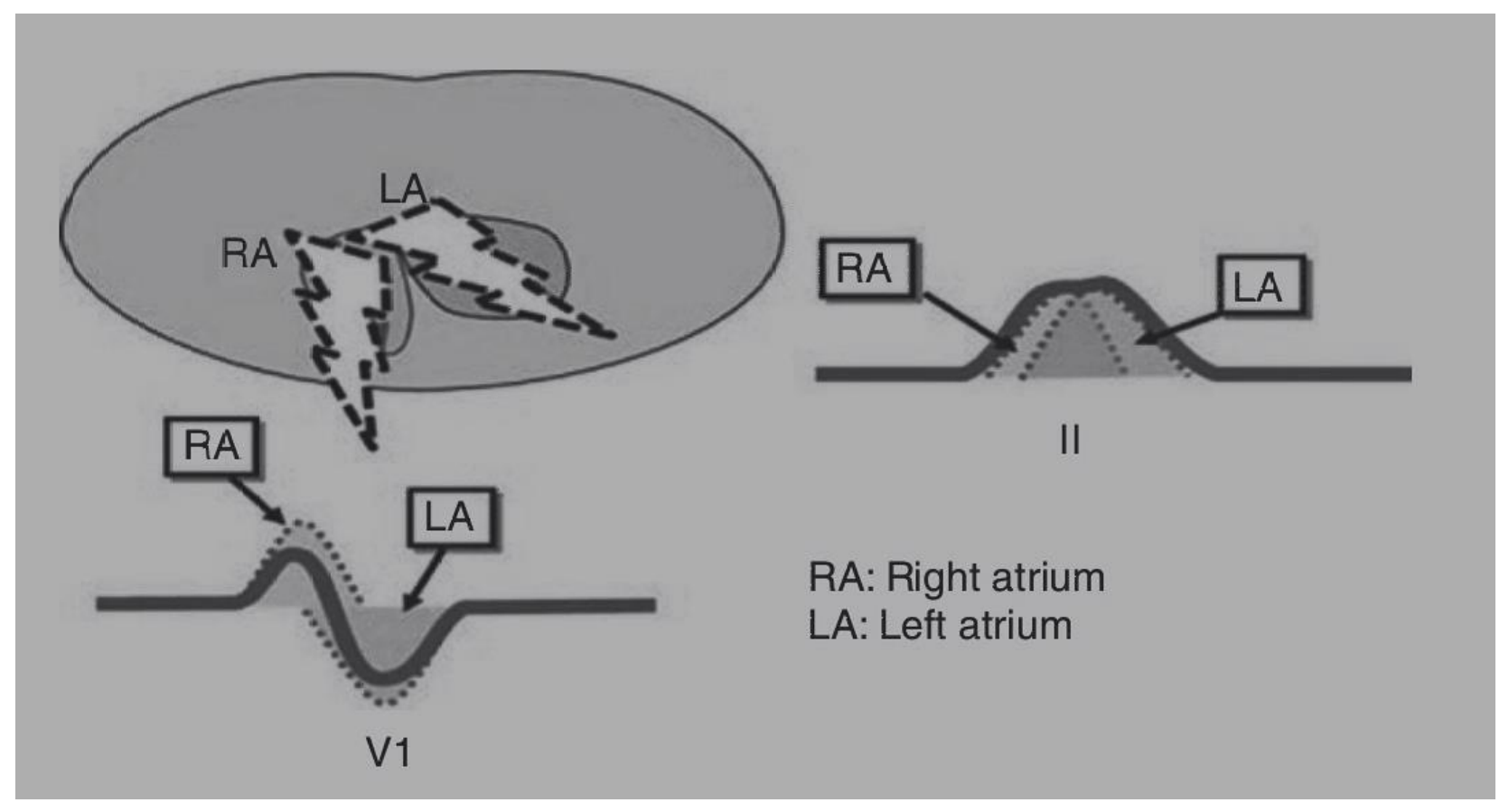

Figure 6. $\mathrm{P}$ wave and its right atrial and left atrial components: initial wave reflects right atrial depolarization, latter part reflects left atrial depolarization

III. If $P$ waves are absent, is there any atrial activity?

1. Sawtooth baseline $\rightarrow$ flutter waves

2. Chaotic baseline $\rightarrow$ fibrillation

3.Flat line $\rightarrow$ no atrial activity at all.
4.P wave in atrial tachycardia. Atrial tachycardia has a more or less regular heart rate $>100 \mathrm{bpm}$, with narrow QRS complexes but $P$-wave(s) originate from another site(s) in the atria (4) 


\section{Step 7: Calculate and evaluate PR interval}

The PR interval is assessed to determine whether impulse conduction from the atria to the ventricles is normal

in terms of speed. A normal PR interval ranges between 120-200 ms (3-5 small squares).

I.A prolonged PR interval suggests the presence of atrioventricular delay (AV block).

II.The shortened PR can mean one of two things:

1. The $P$ wave is originating from somewhere closer to the AV node so the conduction takes less time.

2.The atrial impulse reaches the ventricle by a faster shortcut instead of conducting slowly across the atrial wall.

Example: The Low-Ganong-Levine syndrome is a clinical syndrome consisting of paroxysms of tachycardia and ECG findings of short PR interval and normal QRS duration $(2,3)$.

\section{Step 8: Analyze QRS Complex}

I.When assessing a QRS complex, you need to pay attention to the following characteristics: width, height, morphology

II. The normal duration of the QRS complex measures from: $0.06-0.10$ second.

III. When the QRS measurement is 0.12 seconds or greater it indicates a delay in the electrical impulse as it is passing through the ventricular conduction system.

IV.Q-waves: Isolated $Q$ waves can be normal. A pathological Q wave is $>25 \%$ the size of the $R$ wave that follows it or $>2 \mathrm{~mm}$ in height and $>40 \mathrm{~ms}$ in width.

V.R and $S$ waves: Assess the $R$ wave progression across the chest leads (from small in V1 to large in V6). The transition from $S>R$ wave to $R>S$ wave should occur in V3 or V4. Poor progression (i.e. S $>$ R through to leads V5 and V6) can be a sign of previous MI but can also occur in very large people due to poor lead position.

VI.J point segment: The J point is where the $S$ wave joins the ST segment. This point can be elevated resulting in the ST segment that follows it also being raised (this is known as "high take-off") (2).

\section{Step 9: Analyze ST segment}

The ST segment is the part of the ECG between the end of the $S$ wave and the start of the T wave. In a healthy individual, it should be on an isoelectric line (neither elevated nor depressed). Abnormalities of the ST segment should be investigated to rule out pathology. I.ST-elevation

1. ST-elevation is significant when it is greater than 1 $\mathrm{mm}$ ( 1 small square) in 2 or more contiguous limb leads or $>2 \mathrm{~mm}$ in 2 or more chest leads.

2. It is most commonly caused by acute myocardial infarction (Fig. 7)

II. ST depression

1. ST depression $\geq 0.5 \mathrm{~mm}$ in $\geq 2$ contiguous leads.

2.It indicates myocardial ischemia $(2,3)$.

\section{Step 10: Examine T waves}

$T$ waves represent repolarization of the ventricles.

I.Tall T waves

1.T waves are considered tall if they are: $>5 \mathrm{~mm}$ in the limb leads and $>10 \mathrm{~mm}$ in the chest leads (the same criteria as 'small' QRS complexes)

2. Tall $T$ waves can be associated with hyperkalemia ("tall tented $\mathrm{T}$ waves"), hyperacute ST-elevation myocardial infarction: tall tented $T$ waves, inverted $T$ wave. T waves are normally inverted in $\mathrm{V} 1$ and inversion in lead III is a normal variant.II. Inverted T waves in other leads are a nonspecific sign of a wide variety of conditions: ischemia, bundle branch blocks (V4-6 in left bundle branc block and V1-V3 in right bundle branch block), pulmonary embolism, left ventricular hypertrophy (in the lateral leads), hypertrophic cardiomyopathy (widespread) $(2,3)$.

Step 11: Evaluate QT interval QT interval reflects the total duration of ventricular depolarization and repolarization. It is measured from the onset of the QRS complex to the end of the T-wave. The QT duration is inversely related to heart rate; QT interval increases at slower heart rates and decreases at higher heart rates. Therefore, to determine whether the QT interval is within normal limits, it is necessary to adjust for the heart rate. The heart rate adjusted QT interval referred to as the corrected QT interval (QTc interval). Bazett's formula has traditionally been used to calculate the corrected QT duration.

Normal values for QTc interval is $\leq 0.450 \mathrm{~s}$ for men and $\leq 0.470$ s for women $(2,3)$. 


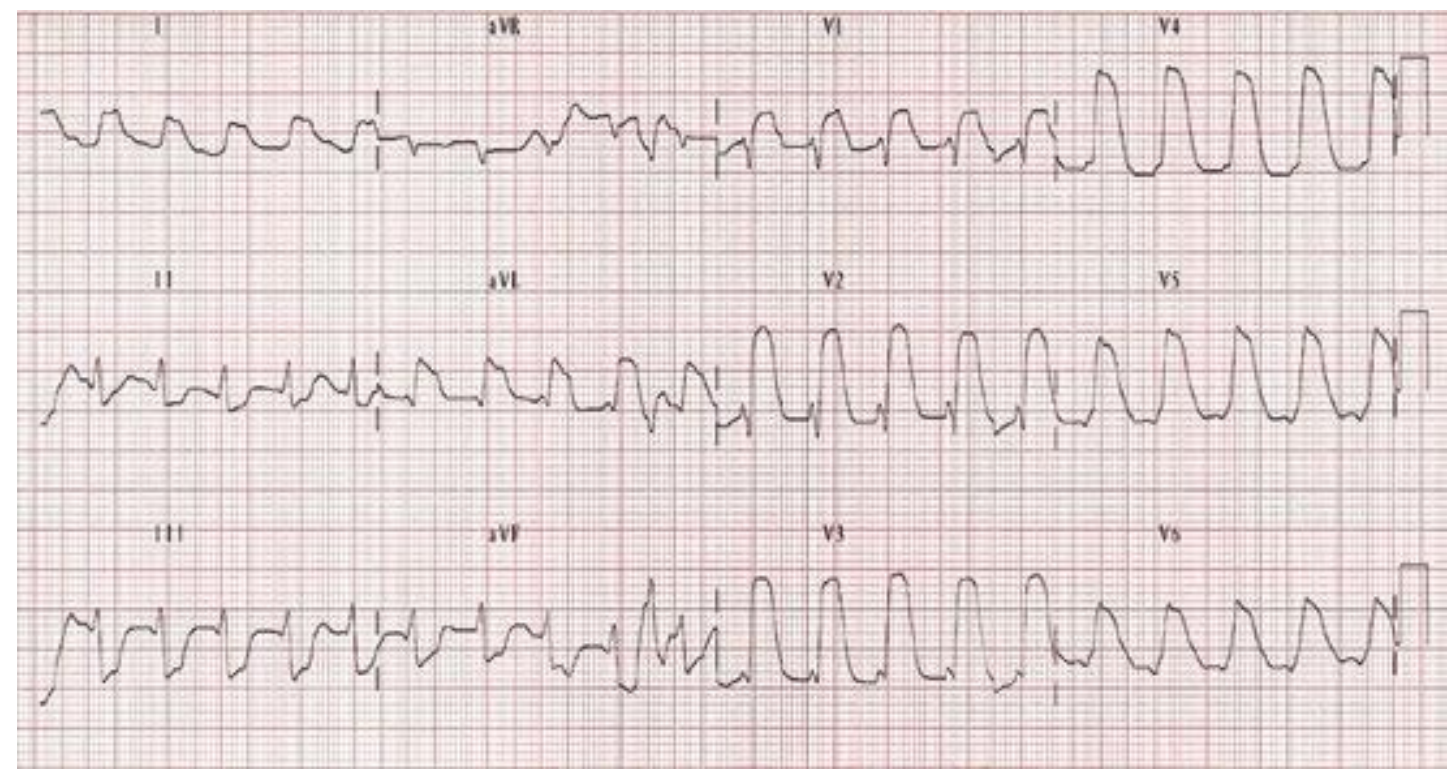

Figure 7. Acute extensive anterior wall myocardial infarction. There are ST segment elevations in V1-V6, I and aVL. Reciprocal ST segment depression can be easily noticed in inferior leads. There is sinus tachycardia with a heart rate of $120 \mathrm{bpm}$. This pattern of ST elevation frequently called as tomb stoning ST elevation.

\section{Step 12: Evaluate $U$ waves}

$U$ waves are not a common finding. The $U$ wave is a > $0.5 \mathrm{~mm}$ deflection after the T wave best seen in V2 or V3. This becomes larger the slower the bradycardia classically $U$ waves are seen in various electrolyte imbalances, hypothermia and secondary to antiarrhythmic therapy (such as digoxin, procainamide or amiodarone).

Peer-review: External and internal Conflict of interest: None to declare Authorship: I.G.

Acknowledgments and funding: None to declare

\section{References}

1. Mattu A, Brady W. ECG's for the Emergency Physician Maiden MA: Blackwell publ; 2013: pp. 176.
2. https://ecgwaves.com/topic/ecg-normal-p-wave-qrscomplex-st-segment-t-wave-j-point/

3. Chan T, Brady W, Harrigan R, Ornato J, Rosen P. ECG in Emergency Medicine and Acute Care. USA: Mosby; 2004; pp. 416. $x$

4. Okutucu S, Oto A. Interpreting ECG in Clinical Practice. Springer; 2018; pp. 119.

5. Rautaharju PM, Surawicz B, Gettes LG, Bailey JJ, Childers R, Deal BJ, et al. AHA/ACCF/HRS recommendations for standardization and interpretation of the electrocardiogram: part IV ST segment, $T$ and $U$ waves, and the $Q T$ interval: a scientific statement from the American Heart Association Electrocardiography and Arrhythmias Committee, Council on Clinical Cardiology; The American College of Cardiology Foundation; and the Heart Rhythm Society. Endorsed by International Society for Computerized Electrocardiology. J Am Coll Cardiol 2009; 53; 982-91 doi: 10.1016/j.jacc.200812.014 


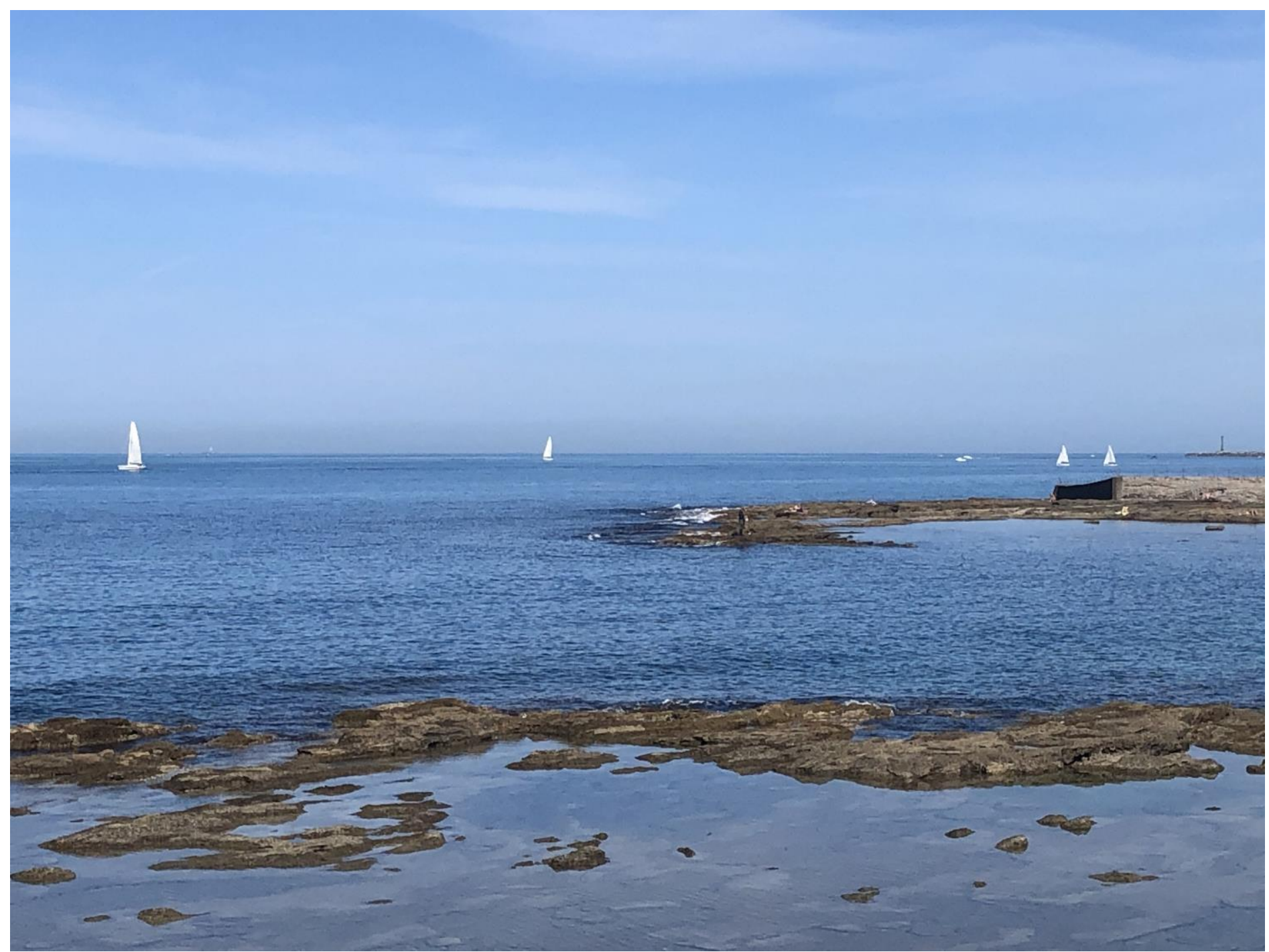

Livorno, Mediterranean Sea, Italy, May 2021. Alessia Gimelli, Italy (Copyright @2021) 